\title{
A!
}

This is an electronic reprint of the original article.

This reprint may differ from the original in pagination and typographic detail.

Nyman, M.; Kivijärvi, V.; Shevchenko, A.; Kaivola, M.

\section{Generation of light in spatially dispersive materials}

Published in:

Physical Review A

DOI:

10.1103/PhysRevA.95.043802

Published: 04/04/2017

Document Version

Publisher's PDF, also known as Version of record

Please cite the original version:

Nyman, M., Kivijärvi, V., Shevchenko, A., \& Kaivola, M. (2017). Generation of light in spatially dispersive materials. Physical Review A, 95(4), 1-9. [043802]. https://doi.org/10.1103/PhysRevA.95.043802

This material is protected by copyright and other intellectual property rights, and duplication or sale of all or part of any of the repository collections is not permitted, except that material may be duplicated by you for your research use or educational purposes in electronic or print form. You must obtain permission for any other use. Electronic or print copies may not be offered, whether for sale or otherwise to anyone who is not an authorised user. 


\title{
Generation of light in spatially dispersive materials
}

\author{
M. Nyman, ${ }^{*}$ V. Kivijärvi, A. Shevchenko, ${ }^{\dagger}$ and M. Kaivola \\ Department of Applied Physics, Aalto University, P.O. Box 13500, FI-00076 Aalto, Finland
}

(Received 5 December 2016; published 4 April 2017)

\begin{abstract}
Spatial dispersion makes optical properties of materials depend on the direction of light propagation. The effect can be applied to control optical emission by sources embedded in such media. We propose a method to determine the radiation pattern of essentially any emitter located in a general spatially dispersive and optically anisotropic medium. The method is based on a decomposition of the source into waves of electric current, each creating optical plane waves whose properties are determined by the wave parameters, the refractive index, and impedance. The method is computationally fast and very accurate even in strongly spatially dispersive plasmonic metamaterials. In particular, we observe large modification of dipole emission in a bifacial and a diffraction-compensating metamaterial. The method is applicable to a large variety of nanostructured materials and, therefore, we believe that it can find numerous applications in nano-optics.
\end{abstract}

DOI: 10.1103/PhysRevA.95.043802

\section{INTRODUCTION}

The properties of photonic emitters, such as fluorescent molecules and quantum dots, are based not only on their intrinsic quantum-mechanical properties, but also on their interaction with the surrounding electromagnetic environment. It is well known that the spontaneous emission rate, directivity of emission, and spectral line shape of an emitter can be modified by placing the emitter in a cavity or near a resonant nanoparticle [1]. However, if the emitter is embedded in a homogeneous material, which is often the case, the surrounding medium also influences the emitter's properties through the near-field interactions with the nearest molecules and through the spatially averaged interaction with the entire medium. The latter includes, in general, both temporal and spatial dispersion as well as optical anisotropy of the medium. Dipole emission has been studied in many different host media, including ordinary dielectrics [2], liquid crystals [3], photonic crystals [4,5], and metamaterials [6-9]. In nanostructured media, such as photonic crystals and metamaterials, the material response can be tailored and used to dramatically modify the emission properties. The use of such media offers interesting possibilities in the design of lasers and other light sources [10-14], detectors [15], and energy conversion devices [16-18].

Various theoretical methods to determine the electromagnetic field of a light source, such as a fluorescent molecule, embedded in an arbitrary medium have been proposed. However, they only determine the field in some specific types of materials, including homogeneous anisotropic materials [19-24], lossless photonic crystals with known Bloch modes [25], and one-dimensional nanomaterials, such as dielectric-metal stacks [26]. More complex media, especially spatially dispersive ones, are out of reach of these methods. An important class of such complex media are optical metamaterials consisting of nontrivial nanoscatterers. While direct numerical simulations can be used to model dipole emission in these materials [27-29], the simulations become

\footnotetext{
*markus.nyman@aalto.fi

†andriy.shevchenko@aalto.fi
}

computationally very intensive and time consuming as the entire nanostructure must be modeled, and the physical picture of metamaterials as effectively homogeneous media [30] is lost. Hence, the prediction, design, and optimization of emission in spatially dispersive materials remain difficult.

In this work, we propose a method to determine the radiation of a source with an arbitrary electric-current distribution in an essentially arbitrary spatially dispersive medium. The method relies on a decomposition of the source into a set of sinusoidal waves of electric current that generate electromagnetic fields. The material, in turn, is described in terms of the plane-wave parameters, refractive index and impedance, determined for each frequency, polarization, and propagation direction in the material, as is usually done in the presence of spatial dispersion. The method can equally well treat pointlike sources, such as elementary quantum emitters [31,32], and extended sources used to generate optical beams. It also enables one to predict the influence of the Purcell effect on the emission of fluorescent molecules or quantum dots embedded in a given medium. When used to study emitters in a nanostructured material, the method provides physical insight into the emission characteristics, which makes designing the material simpler. We demonstrate the accuracy of the method by considering a known example of a point source in a homogeneous anisotropic material. Then, we use the method to design a metamaterial that forces an embedded emitter to radiate in one direction only. Finally, the method is applied to study an emitter embedded in an optical metamaterial designed to eliminate optical diffraction.

The paper is organized as follows: Section II describes the electric-current decomposition method and demonstrates its accuracy by an example. In Sec. III, using the method, we study optical emission in two different spatially dispersive metamaterials. Section IV concludes our work.

\section{ELECTRIC-CURRENT DECOMPOSITION METHOD}

In this section, we introduce a method to determine the radiation of an arbitrary source in a spatially dispersive medium. We consider the problem in time-harmonic form such that all fields and currents oscillate at angular frequency $\omega$ and 


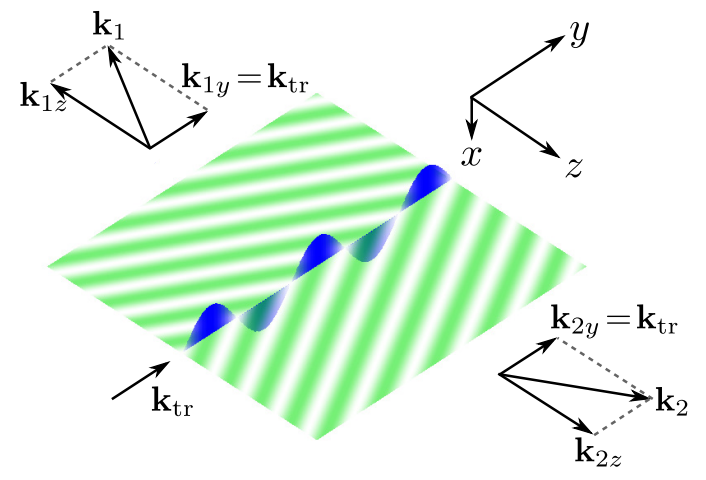

FIG. 1. A wave of electric current (blue) confined to an $x y$ surface propagates in the $y$ direction with a wave vector $\mathbf{k}_{\mathrm{tr}}$ and generates two optical plane waves (green), one to the negative $z$ direction (wave vector $\mathbf{k}_{1}$ ) and one to the positive $z$ direction (wave vector $\mathbf{k}_{2}$ ). The $y$ component of each wave vector is $\mathbf{k}_{\mathrm{tr}}$.

are expressed as $\mathbf{F}(\mathbf{r}, t)=\mathbf{F}(\mathbf{r}) e^{-i \omega t}$, where $\mathbf{F}(\mathbf{r})$ is the complex amplitude of the quantity in question at position $\mathbf{r}$.

\section{A. The principle}

Let us consider a time-harmonic source defined by a current-density distribution $\mathbf{J}(\mathbf{r})$. Without loss of generality, let the source be localized on an $x y$ plane at $z=0$, such that $\mathbf{J}(\mathbf{r})=\mathbf{K}(x, y) \delta(z)$, where $\mathbf{K}(x, y)$ is the surface current density and $\delta(z)$ is the Dirac delta function. Our definition of surface current also includes currents in the direction perpendicular to the surface.

By two-dimensional Fourier transformation, the surface current density can be decomposed into waves of electric current defined on the $x y$ plane. The decomposition is

$$
\mathbf{K}(x, y)=\frac{1}{4 \pi^{2}} \int_{-\infty}^{\infty} \int_{-\infty}^{\infty} \tilde{\mathbf{K}}\left(k_{x}, k_{y}\right) e^{i\left(k_{x} x+k_{y} y\right)} d k_{x} d k_{y},
$$

where $\tilde{\mathbf{K}}\left(k_{x}, k_{y}\right)$ denotes the spatial-frequency spectrum determined by the amplitudes of the current waves with the wave-vector components $k_{x}$ and $k_{y}$. The amplitude spectrum is determined by the Fourier transform

$$
\tilde{\mathbf{K}}\left(k_{x}, k_{y}\right)=\int_{-\infty}^{\infty} \int_{-\infty}^{\infty} \mathbf{K}(x, y) e^{-i\left(k_{x} x+k_{y} y\right)} d x d y .
$$

The reason for decomposing the source into current waves is that each of the waves generates two optical plane waves, propagating in positive and negative $z$ directions while having the same wave-vector components $k_{x}$ and $k_{y}$ as the current wave, as required by the phase-matching condition (see Fig. 1). The interaction of plane waves with the host medium, also in the presence of spatial dispersion, is conveniently described in terms of wave parameters, such as refractive index $n$ and impedance $\eta$ that are specific for each plane wave. Knowing these parameters, we can find the field amplitudes of the generated plane waves and superpose them at any distance from the source, thus finding the radiated field. Note that, for spatially dispersive media, the tensor-form permittivity and permeability-often used when the medium is optically anisotropic - are not helpful, as the tensor elements vary with the propagation angle.
In our calculations, the plane waves propagating in the medium are considered to be transverse. This approximation is usual when retrieving the wave parameters for anisotropic optical metamaterials as it significantly simplifies the problem without compromising much the accuracy of the results [33]. Determining the plane-wave fields created by a current wave then reduces to solving Maxwell's equations in a homogeneous isotropic medium with material parameters equal to the wave parameters. However, if the unit cells of the medium are not centrosymmetric, the wave parameters may be different for the plane waves created on the left and right sides of the current wave $[34,35]$. In such cases, the material parameters on each side must be equated to the corresponding wave parameters.

\section{B. Boundary conditions}

The fields on the two sides of a two-dimensional current wave can be related to each other using electromagnetic boundary conditions that we now derive. Let us consider a current wave with the wave vector along the $y$ axis, described by a surface current density

$$
\mathbf{K}(x, y)=\mathbf{K}_{0} e^{i k_{y} y},
$$

where $\mathbf{K}_{0}$ is the complex amplitude of the current that is independent of $x$. Different sets of boundary conditions are relevant to different vector components of the surface current. Figure 2 illustrates the differing cases with the $x-, y$-, and $z$-polarized currents in Figs. 2(a), 2(b), and 2(c), respectively. The electric and magnetic fields $\mathbf{E}$ and $\mathbf{H}$ on the left and right sides of the boundary are indicated by the subscripts 1 and 2 , respectively. In the picture, field vectors are directed such that their $x, y$, and $z$ components are non-negative. The boundary conditions are obtained by integrating the time-harmonic (a)

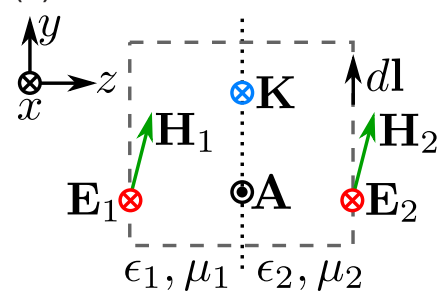

(b)

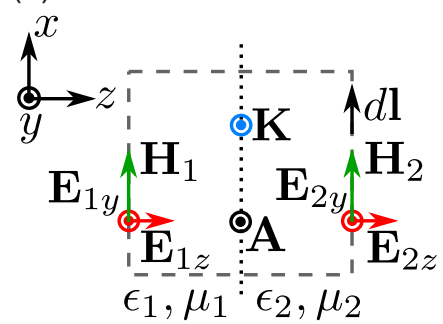

(c)

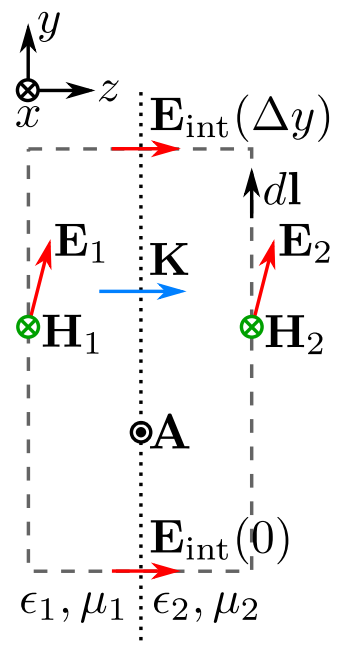

FIG. 2. An interface (dotted line) carries a sinusoidal wave of electric current with a $y$-directional wave vector and an amplitude $\mathbf{K}$. The current is $x, y$, and $z$ polarized in (a), (b), and (c), respectively. A surface area (vector A) delimited by a rectangular contour C (gray dashed line) is used for integrating Maxwell's equations. The relevant electric field $\mathbf{E}$ and magnetic field $\mathbf{H}$ are shown in each picture, and in (c) an additional electric field $\mathbf{E}_{\text {int }}$ internal to the current is present. 
Maxwell equations $\nabla \times \mathbf{E}=i \omega \mu \mathbf{H}$ and $\nabla \times \mathbf{H}=\mathbf{J}-i \omega \epsilon \mathbf{E}$ over the area (vector $\mathbf{A}$ ) delimited by the contour $\mathrm{C}$, shown by the gray dashed line in each panel of Fig. 2, as

$$
\begin{gathered}
\oint_{\mathrm{C}} \mathbf{E} \cdot d \mathbf{l}=i \omega \iint_{\mathrm{A}} \mu \mathbf{H} \cdot d \mathbf{A}, \\
\oint_{\mathrm{C}} \mathbf{H} \cdot d \mathbf{l}=\iint_{\mathrm{A}} \mathbf{J} \cdot d \mathbf{A}-i \omega \iint_{\mathrm{A}} \epsilon \mathbf{E} \cdot d \mathbf{A} .
\end{gathered}
$$

Here, $\epsilon$ and $\mu$ are plane-wave electric permittivity and magnetic permeability, respectively. For the $x$-directional current component, we obtain

$$
\begin{gathered}
E_{2 x}-E_{1 x}=0, \\
H_{2 y}-H_{1 y}=-K_{0 x},
\end{gathered}
$$

while for the $y$-directional component the boundary conditions are

$$
\begin{gathered}
E_{2 y}-E_{1 y}=0, \\
H_{2 x}-H_{1 x}=K_{0 y} .
\end{gathered}
$$

Equations (6) and (8) are the standard boundary conditions because the current density does not affect them, while Eqs. (7) and (9) are derived by integrating in Eq. (5) over the contours shown in Figs. 2(a) and 2(b), respectively. Note that the integration contour used to derive Eq. (9) lies in the $x z$ plane. The case of the $z$-directional current is slightly more involved, as the internal electric field $\mathbf{E}_{\text {int }}$ of the current surface contributes to the contour integration in Eq. (4). The current results in an oscillating electric charge density $\rho_{\mathrm{f}}$ that is described by the continuity equation $i \omega \rho_{\mathrm{f}}=\nabla \cdot \mathbf{J}$, where $\mathbf{J}=\mathbf{K} \delta(z)$. This implies that

$$
\rho_{\mathrm{f}}=\frac{K_{z}}{i \omega} \frac{\partial \delta(z)}{\partial z} .
$$

Since $\nabla \cdot \mathbf{D}=\rho_{\mathrm{f}}$, we obtain

$$
E_{\text {int }}=\frac{D_{\text {int }, z}}{\epsilon}=\frac{K_{0 z}}{i \omega \epsilon} \delta(z) e^{i k_{y} y},
$$

where Eq. (3) was used. Note that $\mathbf{E}_{\text {int }}$ has different phases at the top and the bottom of the integration contour. Hence, Eq. (4) becomes

$$
\begin{aligned}
& \int_{y}^{y+\Delta y}\left(E_{2 y}-E_{1 y}\right) d y \\
& \quad+\int_{-\Delta z / 2}^{\Delta z / 2} \frac{K_{0 z}}{i \omega \epsilon} \delta(z) e^{i k_{y} y}\left(1-e^{i k_{y} \Delta y}\right) d z=0 .
\end{aligned}
$$

In the limits of $\Delta y \rightarrow 0$ and $\Delta z \rightarrow 0$, we use $1-e^{i k_{y} \Delta y}=$ $-i k_{y} \Delta y$ and obtain

$$
E_{2 y}-E_{1 y}=\frac{K_{0 z} k_{y}}{2 \omega}\left(\frac{1}{\epsilon_{1}}+\frac{1}{\epsilon_{2}}\right) e^{i k_{y} y} .
$$

The boundary condition for the magnetic field in this case is standard and given by

$$
H_{2 x}-H_{1 x}=0 .
$$

\section{Field amplitudes}

In order to solve for the amplitudes of the radiated plane waves, we write Maxwell's equations for each of the waves, distinguishing between the transverse-electric (TE) and transverse-magnetic (TM) polarizations. For TE waves, the electric field is $x$ directional [as in Fig. 2(a)], while for TM waves it is in the $y z$ plane [as in Figs. 2(b) and 2(c)]. For TE waves, the equation $\nabla \times \mathbf{E}=i \omega \mu \mathbf{H}$ yields

$$
\begin{gathered}
k_{z} E_{x}=\omega \mu H_{y}, \\
-k_{y} E_{x}=\omega \mu H_{z} .
\end{gathered}
$$

Similarly, using the equation $\nabla \times \mathbf{H}=-i \omega \epsilon \mathbf{E}$, we obtain for TM-polarized waves

$$
\begin{gathered}
k_{z} H_{x}=-\omega \epsilon E_{y}, \\
-k_{y} H_{x}=-\omega \epsilon E_{z} .
\end{gathered}
$$

It is then straightforward to combine the boundary conditions and Eqs. (16)-(19) to determine the amplitudes of the radiated waves. Let us write the electric field amplitudes for the wave radiated to the positive $z$ direction by an $x$-directional current. Equations (6), (7), and (16) give

$$
E_{2 x}=-\left(-\frac{k_{1 z}}{k_{0} n_{1} \eta_{1}}+\frac{k_{2 z}}{k_{0} n_{2} \eta_{2}}\right)^{-1} K_{0 x} e^{i k_{y} y} e^{i k_{z} z} .
$$

We have used the relations $\epsilon=n /(\eta c), \mu=(n \eta) / c$, and $k_{0}=$ $\omega / c$ to switch from $\epsilon$ and $\mu$ to $n$ and $\eta$ as those are the wave parameters we use in our examples. The amplitude of the wave is seen to depend not only on its own wave parameters, but also on the parameters of the wave radiated in the opposite direction. Note also that the real part of $k_{1 z}$ is negative, as the wave in medium 1 propagates to the negative $z$ direction. For the wave generated by a $y$-directional current, Eqs. (8), (9), and (18) yield

$$
E_{2 y}=-\left(-\frac{k_{0} n_{1}}{k_{1 z} \eta_{1}}+\frac{k_{0} n_{2}}{k_{2 z} \eta_{2}}\right)^{-1} K_{0 y} e^{i k_{y} y} e^{i k_{z} z} .
$$

The $z$ component of the electric field is given by $E_{2 z}=$ $-\left(k_{y} / k_{z}\right) E_{2 y}$. For the wave generated by a $z$-directional current, Eqs. (14), (15), and (18) give

$$
E_{2 y}=\left(1-\frac{k_{1 z} \eta_{1} n_{2}}{k_{2 z} \eta_{2} n_{1}}\right)^{-1}\left(\frac{\eta_{1}}{n_{1}}+\frac{\eta_{2}}{n_{2}}\right) \frac{k_{y}}{2 k_{0}} K_{0 z} e^{i k_{y} y} e^{i k_{z} z} .
$$

These equations can be written for a current wave propagating in an arbitrary direction in the $(x, y)$ plane and used in the general form to describe all plane-wave components of any localized surface current [see Eqs. (1) and (2)]. When evaluated for the whole spatial frequency spectrum of a given source, the electric field amplitudes such as those in Eqs. (20)-(22) form the angular spectrum $\tilde{\mathbf{E}}\left(k_{x}, k_{y}\right)$ emitted by the source. The actual electric field at $z=0$ can then be calculated by using the following inverse Fourier transformation:

$$
\mathbf{E}(x, y)=\frac{1}{4 \pi^{2}} \int_{-\infty}^{\infty} \int_{-\infty}^{\infty} \tilde{\mathbf{E}}\left(k_{x}, k_{y}\right) e^{i\left(k_{x} x+k_{y} y\right)} d k_{x} d k_{y} .
$$




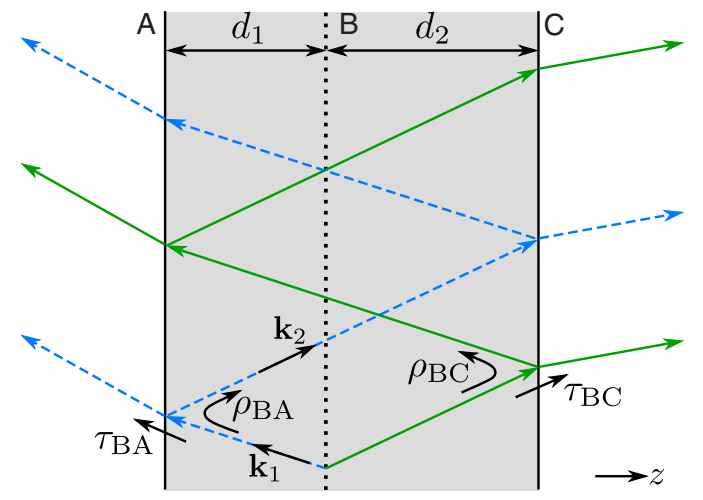

FIG. 3. Calculation of fields created by a source embedded in a metamaterial slab (material B). The slab is surrounded by isotropic materials $\mathrm{A}$ and $\mathrm{C}$ and the source is assumed to be located on the surface shown by the black dotted line. Each plane wave, for which the $\mathbf{k}$-vectors are pictured, experiences multiple reflections from the interfaces. The transmitted and reflected wave amplitudes are given by the generalized Fresnel coefficients $\tau_{\mathrm{BA}}, \rho_{\mathrm{BA}}, \tau_{\mathrm{BC}}$, and $\rho_{\mathrm{BC}}$. Solid green (dashed blue) lines denote plane waves sent by the source to the positive (negative) $z$ direction.

The angular spectrum can be propagated to another location by multiplying it by $\exp \left(i k_{z} z\right)$, after which Eq. (23) gives the field distribution at any $z$.

If the source is embedded in a slab of a spatially dispersive material, it is of practical interest to calculate the field outside the slab where it can be measured. The way to do this is shown schematically in Fig. 3. Let the slab (medium B) be sandwiched between two isotropic media $\mathrm{A}$ and $\mathrm{C}$, e.g., air and some substrate material. Let also the source be located at a distance $d_{1}$ from the interface between media $\mathrm{A}$ and $\mathrm{B}$ and a distance $d_{2}$ from the other interface between B and C. Each plane wave of the generated angular spectrum undergoes multiple reflections at the slab boundaries, and a part of the wave is transmitted out at each reflection. The transmission and reflection coefficients of the interfaces are the generalized Fresnel coefficients that can be calculated from $n$ and $\eta$ [33-35]. When the source sends the angular spectrum $\tilde{E}_{2}\left(k_{x}, k_{y}\right)$ to the positive $z$ direction (solid green arrows in Fig. 3$)$ and $\tilde{E}_{1}\left(k_{x}, k_{y}\right)$ to the negative $z$ direction (dashed blue arrows in Fig. 3), the angular spectra of the field on the left and right surfaces of the slab in media A and C, respectively, are

$$
\begin{aligned}
& \tilde{E}_{\mathrm{L}}=\tau_{\mathrm{BA}} e^{i k_{1 z} d_{1}} \frac{\tilde{E}_{1}+\rho_{\mathrm{BC}} e^{i\left(k_{1 z}+k_{2 z}\right) d_{2}} \tilde{E}_{2}}{1-\rho_{\mathrm{BA}} \rho_{\mathrm{BC}} e^{i\left(k_{1 z}+k_{2 z}\right)\left(d_{1}+d_{2}\right)}}, \\
& \tilde{E}_{\mathrm{R}}=\tau_{\mathrm{BC}} e^{i k_{2 z} d_{2}} \frac{\tilde{E}_{2}+\rho_{\mathrm{BA}} e^{i\left(k_{1 z}+k_{2 z}\right) d_{1}} \tilde{E}_{1}}{1-\rho_{\mathrm{BA}} \rho_{\mathrm{BC}} e^{i\left(k_{1 z}+k_{2 z}\right)\left(d_{1}+d_{2}\right)}} .
\end{aligned}
$$

Here, $\tau_{i j}$ and $\rho_{i j}$ are the generalized Fresnel transmission and reflection coefficients at the interface between media $i$ and $j$. The field exiting the slab will, upon propagation, become the far field from which one can obtain the directivity of the emission and the radiative power. The fields at the position of the source are also easy to find by superposing the reflected waves, which can be used, e.g., to calculate the Purcell factor (describing the modification of spontaneous emission rate) and the Lamb shift (describing the shift of the emission frequency) [32].

\section{Verification}

To verify the electric-current decomposition method, we consider a simple example of the emission of light by a source embedded in a slab of an optically anisotropic lossless medium. Let us assume that the medium is a uniaxial crystal with the extraordinary axis oriented along the $z$ direction. The permittivity tensor is then

$$
\stackrel{\leftrightarrow}{\epsilon}=\left[\begin{array}{ccc}
\epsilon_{x} & 0 & 0 \\
0 & \epsilon_{x} & 0 \\
0 & 0 & \epsilon_{z}
\end{array}\right] .
$$

With respect to the $x y$ plane, the ordinary waves are TE polarized and the extraordinary waves are TM polarized. As a function of the transverse wave-vector components $k_{x}$ and $k_{y}$, the refractive index for the extraordinary waves is

$$
n_{\mathrm{e}}=\sqrt{\frac{\epsilon_{x}}{\epsilon_{0}}+\left(1-\frac{\epsilon_{x}}{\epsilon_{z}}\right) \frac{k_{x}^{2}+k_{y}^{2}}{k_{0}^{2}}},
$$

while the wave impedance is

$$
\eta_{\mathrm{e}}=\frac{n_{\mathrm{e}}}{c \epsilon_{x}} .
$$

This impedance is the transverse impedance which results in the correct generalized Fresnel coefficients $[34,36]$. For the ordinary TE-polarized waves the wave parameters are isotropic, with the refractive index given by $n_{\mathrm{o}}=\sqrt{\epsilon_{x} / \epsilon_{0}}$ and the impedance by $\eta_{0} / n_{0}$, where $\eta_{0}$ is the impedance of vacuum.

Let us now choose $n_{\mathrm{o}}=1.6$ and $n_{\mathrm{e}}=2.1$, as for a slab of a highly anisotropic, homeotropically oriented bistolane liquid crystal (5OMeO5, see Ref. [37]) and consider a $y$-polarized dipole emitter at the center of the slab. The material outside the crystal is assumed to have a refractive index of 1.6. We also choose the slab thickness to be equal to $\lambda_{0} /\left(2 n_{\mathrm{o}}\right)$, where $\lambda_{0}$ is the vacuum wavelength of the dipole radiation. Using both the electric-current decomposition method and the finiteelement method (FEM) of the commercial software COMSOL MULTIPHYSICS, we compute the electric field on a sphere with radius $r=1.6 \lambda_{0}$ centered on the dipole. At this distance from the source, the field is already nearly of pure far-field type. Figure 4(a) shows two cross sections of the setup. The green and blue circles are the intersections of the sphere with the $x z$ and $y z$ planes, respectively. Figure 4(b) shows the polar plots of the normalized intensities measured on these circles as a function of the polar angle $\theta$ [shown also in Fig. 4(a)]. The solid and dashed lines show the results calculated by the current decomposition method and FEM, respectively. The results are nearly identical, implying that the decomposition method is remarkably accurate. The angles close to $\theta=90^{\circ}$ and $\theta=270^{\circ}$ are omitted from the calculation because the part of the sphere corresponding to them lies inside the slab.

The problem of dipole radiation in optically anisotropic layered media without spatial dispersion has been previously considered using the Green function formalism [19-24]. When using the formalism, the materials are described by single electric permittivity and magnetic permeability tensors. However, as soon as spatial dispersion becomes significant, 
(a)
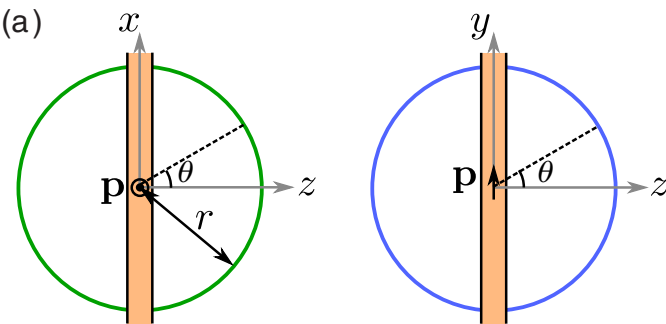

(b)

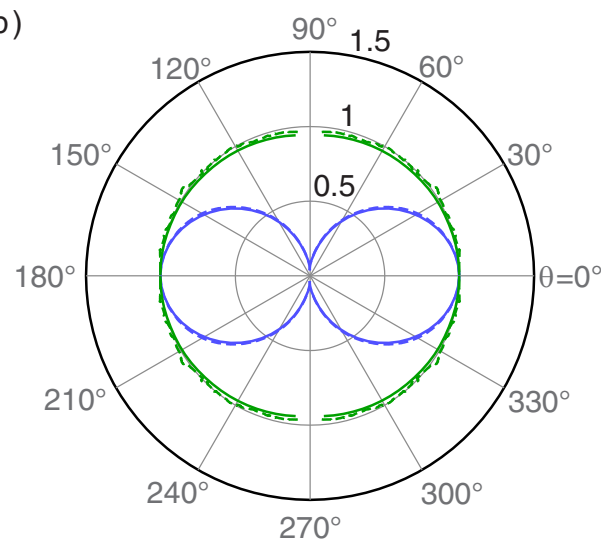

FIG. 4. A $y$-polarized dipole source (dipole moment $\mathbf{p}$ ) is embedded in a slab of a uniaxial anisotropic medium (orange). The emitted intensity distribution of the source is measured on a sphere. (a) The green and blue lines are intersections of this sphere with the $x z$ and $y z$ planes, respectively. (b) The normalized intensity calculated on the green and blue lines is plotted in the corresponding color as a function of the angle $\theta$ shown in (a). The solid and dashed lines show the results of calculations by the electric-current decomposition method and FEM, respectively.

these tensors become impractical due to their dependence on the wave-propagation direction. Then, the fields must be expressed as integrals over the plane-wave modes of the medium, which will make the description analogous to the one presented here. Despite the superficial similarity, no method based on Green functions and suitable for spatially dispersive materials has been presented so far. Our method is developed to be readily applicable to both anisotropic and spatially dispersive media. In the next section, we also show that it is remarkably accurate even in the case of highly complex media.

\section{SPATIALLY DISPERSIVE METAMATERIALS}

In optical metamaterials, spatial dispersion is almost always present because the structural units of the materials have a non-negligible size compared to the optical wavelength. Our method, capable of taking the spatial dispersion into account, is therefore well suited to such materials. Below, we apply the theory to two interesting metamaterial examples.

\section{A. Bifacial metamaterial}

Ordinarily, a pointlike source located inside a homogeneous medium radiates an equal amount of power in opposite directions, because the environment is symmetric. The radiation can be directed into one direction only if the symmetry of the emitter's surroundings is broken, e.g., if it is placed next to a mirror or coupled to a nanoantenna. One might then also expect that breaking the symmetry of the surrounding medium itself could make the emission one-directional. Using our theory, we now examine what kind of a material is required to achieve this novel effect.

Let us consider a single plane-wave component generated by a $y$-polarized emitter and the wave propagating in the positive $z$ direction $\left(k_{x}=k_{y}=0\right)$. For this component, Eq. (21) yields

$$
E_{2 y}=-\frac{J_{0 y}}{1 / \eta_{1}+1 / \eta_{2}} .
$$

Now, considering that the magnetic field amplitude is $H_{2 x}=$ $-E_{2 y} / \eta_{2}$, we can write the time-averaged Poynting vector for the wave as

$$
S_{2}=\frac{1}{2}\left|E_{2 y}\right|^{2} \operatorname{Re}\left\{\frac{1}{\eta_{2}}\right\} .
$$

The source also generates a plane-wave component propagating in the opposite direction (negative $z$ direction). We can easily find that $\left|E_{1 y}\right|=\left|E_{2 y}\right|$ for this wave, and write the Poynting vector as

$$
S_{1}=-\frac{1}{2}\left|E_{1 y}\right|^{2} \operatorname{Re}\left\{\frac{1}{\eta_{1}}\right\} .
$$

It is now obvious that if the impedance $\eta$ is different for the two counterpropagating plane waves, the powers in these waves will also differ. Therefore, a strongly bifacial material is required to force emission only in one direction.

Let us consider an example of a bifacial metamaterial composed of gold nanodimers [34,38]. Figure 5(a) shows the structure of a single layer of the metamaterial. Each metamolecule comprises a larger and a smaller disk with diameters of 110 and $55 \mathrm{~nm}$, respectively, and a thickness of $15 \mathrm{~nm}$. The surface-to-surface separation of the disks is $70 \mathrm{~nm}$. The dimers form a cubic lattice with lattice parameters $\Lambda_{x}=\Lambda_{y}=\Lambda_{z}=180 \mathrm{~nm}$. A wave propagating in the material will excite the two disks of each metamolecule with a non-negligible phase delay, which creates a combined dipole-quadrupole excitation that scatters light unequally in opposite directions. Therefore, the optical response of the material is different for waves propagating in the positive and negative $z$ directions.

We determined the wave parameters $n$ and $\eta$ using a technique described in Ref. [34]. Figure 5(b) shows the refractive index spectrum for waves propagating along the $z$ axis independently of the direction. The impedance spectrum shown in Fig. 5(c) clearly exhibits spatial dispersion, as the spectra are different for waves propagating in the positive and negative $z$ directions (see the red and blue curves). Next, in Fig. 6(a), we plot the spectrum of the factor $\operatorname{Re}\{1 / \eta\}$ present in Eq. (30). At a wavelength of $900 \mathrm{~nm}$ the factor is large for the negative and very small for the positive $z$ direction of wave propagation. We therefore predict that most of the power of an emitter located inside this material will be radiated in the negative $z$ direction. To verify this prediction, we consider a two-layer slab of the material and place a $y$-polarized dipole emitter at the center of the slab. Figure 6(b) shows the intensity distribution created by the emitter (black dot in the center) located in the plane $x=0$. This plane lies 
(a)

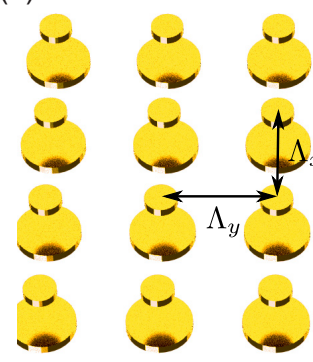

(b)

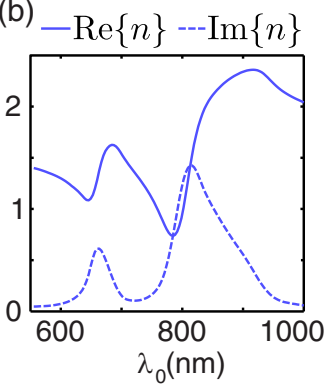

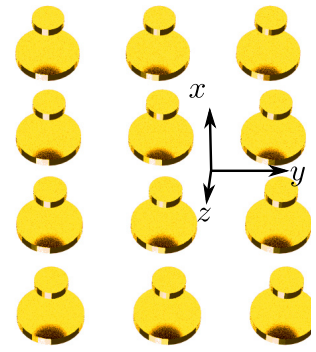

(c) $=\operatorname{Re}\{\eta\}$---- $\operatorname{Im}\{\eta\}$

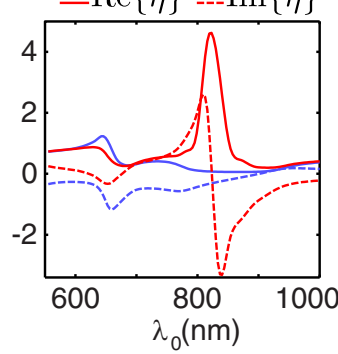

FIG. 5. (a) A bifacial metamaterial composed of an array of gold disk dimers. (b) The spectrum of the effective refractive index $n$ of a wave propagating in the $z$ direction. The blue solid and dashed curves represent the real and imaginary parts of $n$ for waves propagating along the $z$ axis. (c) The spectrum of the effective impedance $\eta$ for a wave propagating in the positive (negative) $z$ direction by red (blue) curves; the solid and dashed lines correspond to the real and imaginary parts, respectively. The values of $\eta$ are normalized to the impedance of vacuum.

(a) 15
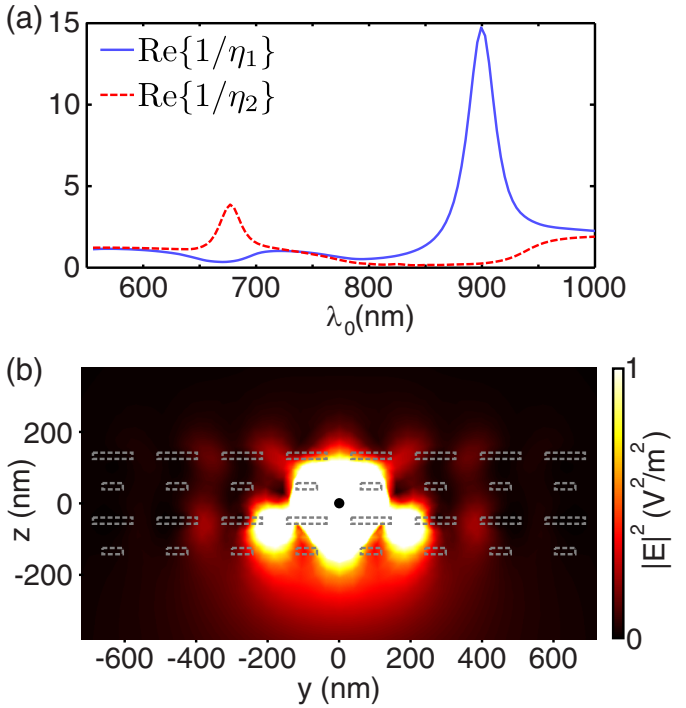

FIG. 6. (a) The factors $\operatorname{Re}\left\{1 / \eta_{1}\right\}$ and $\operatorname{Re}\left\{1 / \eta_{2}\right\}$ corresponding to the negative and positive $z$ direction of the wave propagation in the bifacial metamaterial. (b) The intensity distribution at $x=0$ created by a $y$-polarized emitter (black dot) located between two metamolecular layers of the bifacial metamaterial. At a wavelength of $\lambda_{0}=900 \mathrm{~nm}$, almost all the power is radiated into the negative $z$ direction. The projections of the metamolecules onto the $x=0$ plane are shown by gray lines.

between the rows of the dimers. To visualize the material's structure, the metamolecules are projected onto this plane (see the gray dashed lines). The field distribution was calculated directly with COMSOL MULTIPHYSICS. Clearly, almost all of the radiation is emitted downwards as expected. Comparing the values of intensity on the $z$ axis, we find that the intensity at the bottom is approximately 10 times higher than the intensity at the top. While Fig. 6(a) predicts a larger difference, it does not take into account the internal reflections within the slab. In a more accurate treatment, the reflections can be taken into account using Eqs. (24) and (25). This yields a value of 11 for the intensity ratio, matching well the numerical result.

\section{B. Diffraction-compensating metamaterial}

Let us now consider an example of a metamaterial designed to eliminate optical diffraction. Beams of light propagating in such a material do not diverge. Therefore, a field radiated by a point emitter set inside the material can be expected to propagate in a collimated beam instead of spreading as a spherical wave. A similar effect has in fact previously been considered in the context of self-collimating photonic crystals using direct numerical calculations $[39,40]$. In those studies diffraction and boundary reflection hampered the efficiency of the structures, requiring, e.g., surface modifications. In the metamaterial considered here, these problems are absent due to the impedance matching afforded by the material structure itself and the absence of Bragg reflection effects.

The metamaterial we consider has been studied previously for its image transfer capability [41]. It is composed of a lattice of silver nanorods in glass [see Fig. 7(a)]. By optimizing the structural units, the material's anisotropy and spatial dispersion have been tuned such that the isofrequency contour of the wave vector $\mathbf{k}$ has a flat region at small propagation angles $\theta$, which leads to the elimination of optical diffraction for beams propagating in the $z$ direction. Figures 7(b) and 7(c) show isofrequency contours of the wave parameters $n$ and $\eta$ as functions of $\theta$ for TM polarization at a vacuum wavelength of $\lambda_{0}=790 \mathrm{~nm}$. Only two-dimensional contours are shown instead of surfaces as the parameters are azimuthally symmetric [about the $z$ axis corresponding to $\theta=0$ in Figs. 7(b) and 7(c)]. The contour of $n$ is nearly perfectly flat for $\theta \in\left[-30^{\circ}, 30^{\circ}\right]$. The imaginary part of $n$ is very small in this region, e.g., $\operatorname{Im}\{n\}=0.0015$ at $\theta=0$, which shows that absorption losses are low and do not remarkably affect diffraction compensation. Finally, the impedance is well matched to the impedance of glass around $\theta=0$, which means that reflection losses are also small for all but the most obliquely incident waves. We used an interferometric approach $[34,35,42]$ to retrieve the refractive index and impedance for all relevant waves.

First we demonstrate the action of the metamaterial by considering an infinite array of $y$-polarized coherently radiating electric dipoles stacked in the $x$ direction, one inside each unit cell of the material. This configuration approximately corresponds to a practically realizable situation where the nanomaterial is fabricated into a slab waveguide with a single layer of nanorods that confines the fields in the $x$ 
(a)

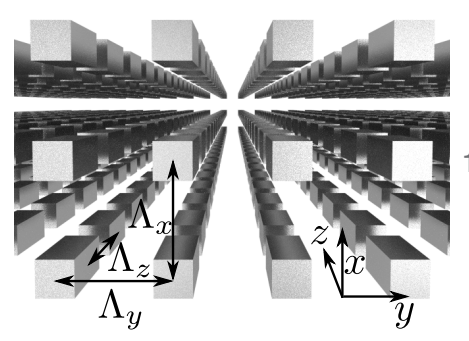

(b)

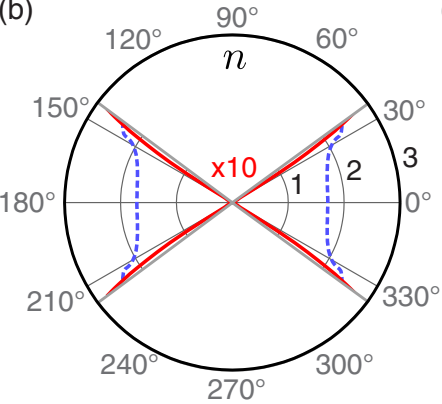

(c)

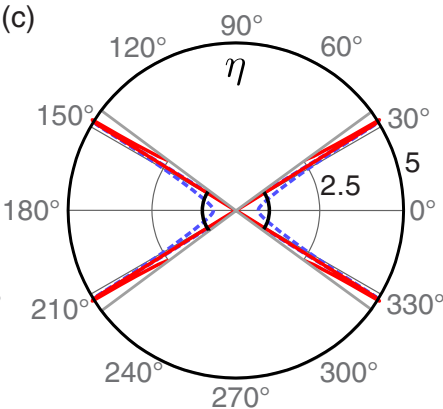

FIG. 7. A diffraction-compensating metamaterial. The material's structure shown in (a) is a lattice of silver nanorods in glass. The lattice constants are $\Lambda_{x}=\Lambda_{y}=120 \mathrm{~nm}$ and $\Lambda_{z}=200 \mathrm{~nm}$. The rod dimensions are $40 \mathrm{~nm}$ in the $x$ and $y$ directions and $130 \mathrm{~nm}$ in the $z$ direction. The material operates at the vacuum wavelength $\lambda_{0}=790 \mathrm{~nm}$. At this wavelength, isofrequency contours of the wave parameters $n$ and $\eta$ are shown in (b) and (c), respectively, as functions of the propagation angle $\theta$ with respect to the $z$ axis for the TM polarization. The blue dashed and red solid curves show respectively the real and imaginary parts of each quantity. In (b) the imaginary part is multiplied by 10 to make it more visible, and in (c), the black curve shows the impedance of glass. The white sectors correspond to angles for which total internal reflection prevents transmission out of the material into the surrounding glass.

direction, allowing them to freely vary only in two dimensions. Figure 8(a) shows the resulting intensity distribution on a $y z$ plane at $x=0$ calculated directly with COMSOL MULTIPHYSICS for an eight-cell-long metamaterial. The position of the source
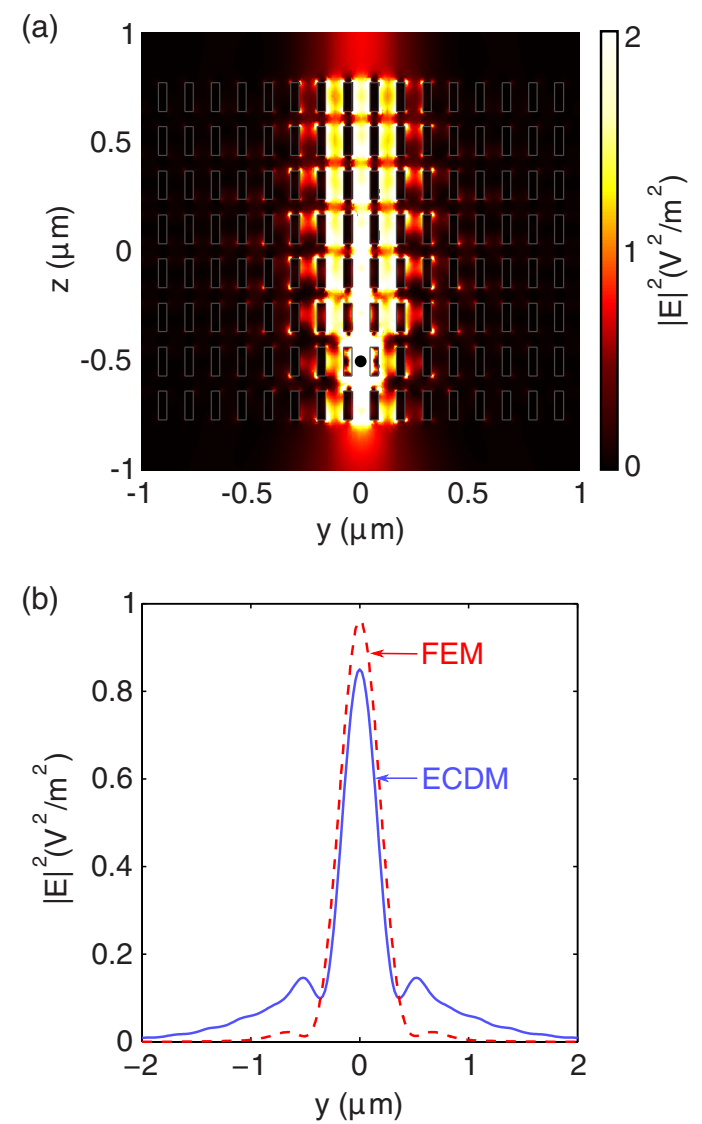

FIG. 8. Radiation of an infinite chain of $y$-polarized dipoles embedded in a slab of a diffraction-compensating metamaterial. The chain is parallel to the $x$ axis. The intensity distribution in the $(y, z)$ plane containing one of the dipoles is shown in (a); the location of the dipole is marked by a black dot. The intensity distribution at $z=1 \mu \mathrm{m}$ is shown in (b). The electric-current decomposition method and FEM results are shown by the blue solid and red dashed lines, respectively. is shown with a black dot at $(y, z)=(0,-0.5)$. The radiation is seen to form a surprisingly well-collimated and narrow optical beam. The intensity profile at the output of the slab (at $z=1 \mu \mathrm{m}$ ) is shown by the red dashed line in Fig. 8(b). We have calculated the same output intensity profile by using our current-decomposition method and obtained the blue solid curve in Fig. 8(b). This curve is nearly identical to the one obtained purely numerically, implying a good accuracy of our method. A small difference is seen in the peak value and in the tails of the profiles. The main reason for this difference is that the near-field coupling of the source to the nearest metamolecules is taken into account only in the direct numerical calculations.

Next we turn to a full three-dimensional calculation of radiation by a single dipole that is a good model of, e.g., a fluorescent molecule. We now position the source between two neighboring layers at the center of the slab of Fig. 8(a) (to $z=0$ ). As previously, we first compute the intensity distribution outside the same slab as in Fig. 8(a) with the FEM and obtain the two-dimensional intensity profile at $z=1 \mu \mathrm{m}$ shown in Fig. 9(a). The bright spot at the center is slightly elongated in the $y$ direction due to the fixed orientation of the dipole along the $y$ axis. Outside the central spot, however, the field spreads more in the $x$ direction than in the $y$ direction. The same features are obtained in our semianalytical calculations, the result of which is shown in Fig. 9(b). For comparison, we have also calculated the radiation pattern at $z=1 \mu \mathrm{m}$ for a dipole in glass [see Fig. 9(c)]. The metamaterial obviously makes the dipole radiation highly directive, with a much higher intensity at the center compared to that in glass. Figure 9(d) shows cross sections of the normalized intensity distributions on a $y$-directional line crossing the center of the beams. In this picture we see more clearly that the FEM (dashed red line) and current-decomposition (solid blue line) results match almost perfectly; the normalized intensity distribution of the dipole radiation in glass is also shown in the figure with a green dotted line. This example therefore proves the validity of our fast semianalytical method in designing truly three-dimensional metamaterial host media for typical dipolar light sources, such as fluorescent molecules and quantum dots. 
(a)

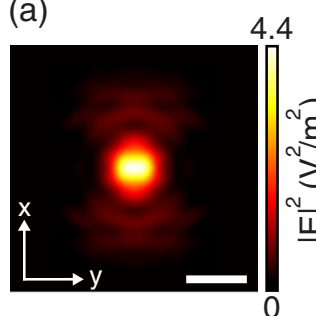

(c)

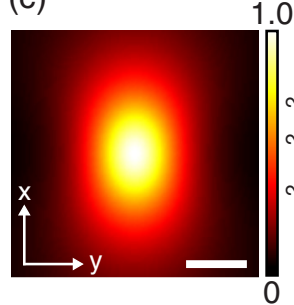

(b)

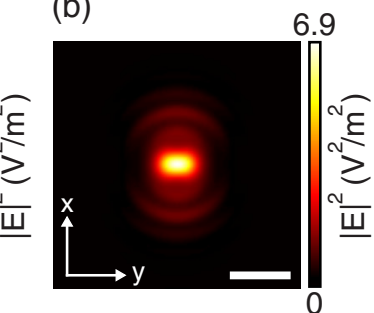

(d) (a)

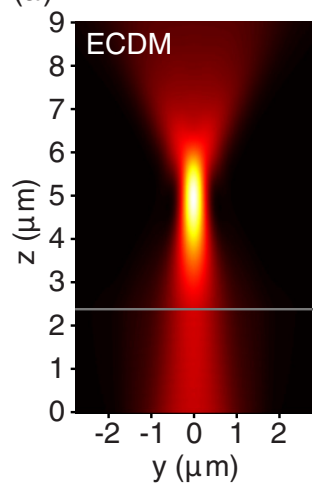

(c)

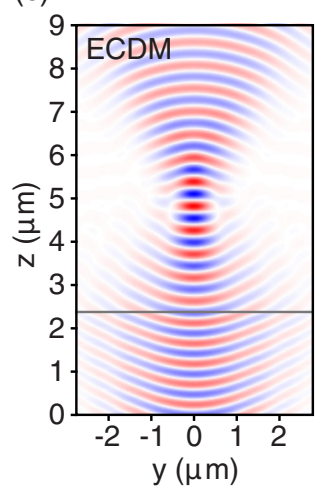

(b)

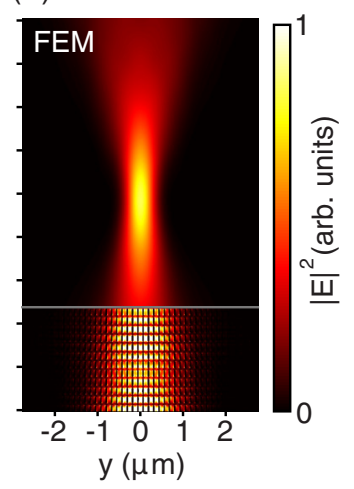

(d)

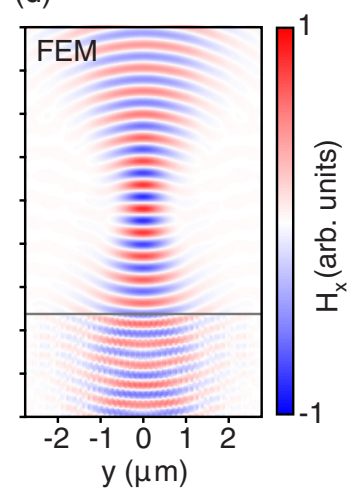

FIG. 9. Output intensity distributions created by a $y$-polarized dipole positioned inside a diffraction-compensating metamaterial slab of Fig. 8(a) at $z=0, y=0$. The distribution is calculated at $z=1 \mu \mathrm{m}$. The results of (a) the FEM and (b) the current decomposition method are shown; (c) shows the intensity at the same distance from the dipole in pure glass. In each picture, the scale bar corresponds to a length of $1 \mu \mathrm{m}$. In (d), the curves show cross sections of the normalized intensity distributions along a $y$-directional line. The dashed red, solid blue, and dotted green curves show the results of the FEM, the current decomposition, and the dipole-in-glass calculations, respectively.

As a final example, we demonstrate the generation of light by an extended source embedded in the same diffractioncompensating metamaterial. We choose the source to have a $y$-polarized electric current distribution that is Gaussian along the $y$ axis. The source is infinitely extended along the $x$ axis to make the calculations effectively two dimensional. We also set the phase distribution of the source such that the generated beam has converging wave fronts. This problem is relevant when, e.g., the radiation is created by scatterers illuminated with a converging, two-dimensional Gaussian beam. In Fig. 10, the beam source is located at $z=0$ inside a semi-infinite material that has 12 metamolecular layers in the positive $z$ direction. Figures 10(a) and 10(b) show the intensity distribution as calculated by our current-decomposition method and FEM, respectively. Similarly, Figs. 10(c) and 10(d) show the magnetic field at a fixed instant of time. The magnetic field is $x$-directed everywhere for this polarization. The slab boundary is shown with a gray line. The transverse intensity distribution created inside the slab is preserved while the beam propagates through the metamaterial in spite of the fact that the beam wave fronts are curved. The transverse phase distribution is also preserved, as can be seen in Figs. 10(c) and 10(d). The beam is focused only when it enters glass that does not compensate for diffraction. Outside the metamaterial, the difference of the semianalytical and full numerical calculation is again small. Inside the slab, the microscopic structure of the material is visible in the FEM results, showing the highintensity near fields of the metamolecules that are absent in the semianalytical calculations. This example not only illustrates an interesting optical effect with a remarkable accuracy, but
FIG. 10. A beam generated by an electric-current density at $z=0$ having a Gaussian amplitude distribution and a phase profile corresponding to a converging spherical wave. The intensity distributions of the generated beam computed by (a) the current decomposition method and (b) the FEM are shown; (c) and (d) show the magnitude of the magnetic field at a fixed instant of time corresponding to the profiles in (a) and (b), respectively. The gray line shows the edge of the metamaterial slab. In (b) and (d), the structure of the material can be observed.

also demonstrates a way to generate an extended light source inside a metamaterial that is independent of the exact technique used. The electric-current decomposition can be utilized in direct numerical calculations as well to define a source.

\section{CONCLUSIONS}

We proposed a semianalytical method to calculate optical fields produced by light sources in a spatially dispersive host medium and verified it numerically by several examples. The examples include point and distributed sources in optically anisotropic and spatially dispersive nanostructured media. In the calculation procedure, any source of interest is decomposed into electric current waves that radiate optical plane waves inside the material. The radiated waves are described by the wave parameters, the refractive index and impedance, that fully characterize any spatially dispersive medium. A set of equations is used to evaluate the complex amplitudes of these plane waves and to find the total field of the source by coherently summing the obtained plane-wave components.

The method provides a clear physical picture for light generation and propagation in the presence of spatial dispersion and is computationally fast, which facilitates its practical use. 
In particular, it allows one to design nanostructured media and optimize their properties in a short computational time. Our two examples demonstrate successful achievement of optical radiation control in a bifacial and a diffraction-compensating metamaterial. A bifacial material was shown to lead to an enhanced directivity of a dipole emitter. We also demonstrated surprising, well-collimated, dipole radiation in the diffractioncompensating metamaterial. Finally, we proposed a way to generate and study arbitrary optical beams inside nanostructured media in both purely numerical and semianalytical calculations. Since essentially all nanostructured materials are spatially dispersive, the method can find wide-range applications in nano-optics.

\section{ACKNOWLEDGMENT}

V.K. acknowledges financial support from the Vilho, Yrjö and Kalle Väisälä Foundation of the Finnish Academy of Science and Letters.
[1] M. Pelton, Nat. Photon. 9, 427 (2015).

[2] W. L. Barnes, J. Mod. Opt. 45, 661 (1998).

[3] H. Shi, B. M. Conger, D. Katsis, and S. H. Chen, Liq. Cryst. 24, 163 (1998).

[4] P. Lodahl, A. van Driel, I. Nikolaev, A. Irman, K. Overgaag, D. Vanmaekelbergh, and W. Vos, Nature (London) 430, 654 (2004).

[5] C. López, Adv. Mater. 15, 1679 (2003).

[6] A. Poddubny, I. Iorsh, P. Belov, and Y. Kivshar, Nat. Photon. 7, 948 (2013).

[7] D. Costantini, A. Lefebvre, A.-L. Coutrot, I. Moldovan-Doyen, J. P. Hugonin, S. Boutami, F. Marquier, H. Benisty, and J.-J. Greffet, Phys. Rev. Appl 4, 014023 (2015).

[8] A. Alù, M. G. Silveirinha, A. Salandrino, and N. Engheta, Phys. Rev. B 75, 155410 (2007).

[9] E. J. R. Vesseur, T. Coenen, H. Caglayan, N. Engheta, and A. Polman, Phys. Rev. Lett. 110, 013902 (2013).

[10] N. I. Zheludev, S. L. Prosvirnin, N. Papasimakis, and V. A. Fedotov, Nat. Photon. 2, 351 (2008).

[11] S. Fan, P. R. Villeneuve, J. D. Joannopoulos, and E. F. Schubert, Phys. Rev. Lett. 78, 3294 (1997).

[12] J. J. Wierer, Jr., A. David, and M. M. Megens, Nat. Photon. 3, 163 (2009).

[13] O. Hess, J. B. Pendry, S. A. Maier, R. F. Oulton, J. M. Hamm, and K. L. Tsakmakidis, Nat. Mater. 11, 573 (2012).

[14] A. K. Sarychev and G. Tartakovsky, Phys. Rev. B 75, 085436 (2007).

[15] P. Alivisatos, Nat. Biotechnol. 22, 47 (2004).

[16] Y. Guo, C. L. Cortes, S. Molesky, and Z. Jacob, Appl. Phys. Lett. 101, 131106 (2012).

[17] X. Liu, T. Tyler, T. Starr, A. F. Starr, N. M. Jokerst, and W. J. Padilla, Phys. Rev. Lett. 107, 045901 (2011).

[18] S. Y. Lin, J. Moreno, and J. G. Fleming, Appl. Phys. Lett. 83, 380 (2003).

[19] J. A. Kong, Geophysics 37, 985 (1972).

[20] M. Memarian and G. V. Eleftheriades, Prog. Electromagn. Res. 142, 437 (2013).

[21] Y. Huang, Ph.D. thesis, Syracuse University, 2014.

[22] S. R. J. Brueck, IEEE J. Sel. Top. Quantum Electron. 6, 899 (2000).

[23] K. Sainath, F. L. Teixeira, and B. Donderici, Phys. Rev. E 89, 013312 (2014)
[24] L. A. Pazynin, S. Sautbekov, Y. K. Sirenko, A. A. Vertiy, and N. P. Yashina, Telecommun. Radio Eng. (Engl. Transl.) 74, 1039 (2015).

[25] R. C. McPhedran, L. C. Botten, J. McOrist, A. A. Asatryan, C. M. de Sterke, and N. A. Nicorovici, Phys. Rev. E 69, 016609 (2004).

[26] X. Ni, G. V. Naik, A. V. Kildishev, Y. Barnakov, A. Boltasseva, and V. M. Shalaev, Appl. Phys. B 103, 553 (2011).

[27] T. Galfsky, H. N. S. Krishnamoorthy, W. Newman, E. E. Narimanov, Z. Jacob, and V. M. Menon, Optica 2, 62 (2015).

[28] D. Lu, J. J. Kan, E. E. Fullerton, and Z. Liu, Nat. Nanotechnol. 9, 48 (2014).

[29] L. Ferrari, D. Lu, D. Lepage, and Z. Liu, Opt. Express 22, 4301 (2014).

[30] A. Andryieuski, S. Ha, A. A. Sukhorukov, Y. S. Kivshar, and A. V. Lavrinenko, Phys. Rev. B 86, 035127 (2012).

[31] L. Novotny and B. Hecht, Principles of Nano-Optics (Cambridge University Press, Cambridge, UK, 2006).

[32] P. Yao, C. Van Vlack, A. Reza, M. Patterson, M. M. Dignam, and S. Hughes, Phys. Rev. B 80, 195106 (2009).

[33] C. Menzel, C. Rockstuhl, T. Paul, F. Lederer, and T. Pertsch, Phys. Rev. B 77, 195328 (2008).

[34] P. Grahn, A. Shevchenko, and M. Kaivola, Opt. Express 21, 23471 (2013).

[35] A. Shevchenko, P. Grahn, and M. Kaivola, J. Nanophotonics 8, 083074 (2014).

[36] A. G. Shalashov and E. D. Gospodchikov, Phys. Usp. 54, 145 (2011).

[37] D. Węgłowska, P. Kula, and J. Herman, RSC Adv. 6, 403 (2015).

[38] A. Shevchenko, V. Kivijärvi, P. Grahn, M. Kaivola, and K. Lindfors, Phys. Rev. Appl. 4, 024019 (2015).

[39] W. Y. Liang, J. W. Dong, and H. Z. Wang, Opt. Express 15, 1234 (2007).

[40] G. T. Reed, Silicon Photonics: The State of the Art (John Wiley \& Sons, New York, 2008).

[41] V. Kivijärvi, M. Nyman, A. Shevchenko, and M. Kaivola, Opt. Express 24, 9806 (2016).

[42] P. Grahn, A. Shevchenko, and M. Kaivola, New J. Phys. 15, 113044 (2013). 